J. Lake Sci. (湖泊科学), 2015, 27 (6): 1059-1066

DOI 10. $18307 / 2015.0610$

(C) 2015 by Journal of Lake Sciences

\title{
经低温、低光处理的藻类在不同光强、氮磷浓度下的生理生态特征”
}

周起超 ${ }^{1,2}$, 熊 倩 ${ }^{1,3}$, 李 林 $^{1}$, 胡 丽 $^{\text {丽 }}{ }^{1,3}$, 宋立荣 ${ }^{1 * *}$

( 1 : 中国科学院水生生物研究所淡水生态与生物技术国家重点实验室, 武汉 430072 )

(2: 云南省环境科学研究院 (中国昆明高原湖泊国际研究中心) 高原湖泊流域污染过程与管理云南省重点实验室, 昆明 $650034)$

(3: 中国科学院大学,北京 100049)

摘 要: 通过低温、低光处理太湖藻类后, 研究光强、营养盐对水华早期藻类生长与组成的影响. 结果显示: 中光强 $\left(50 \mu \mathrm{E} /\left(\mathrm{m}^{2} \cdot \mathrm{s}\right)\right)$ 下藻类生长状态最优, 高光强 $\left(200 \mu \mathrm{E} /\left(\mathrm{m}^{2} \cdot \mathrm{s}\right)\right)$ 次之, 低光强 $\left(5 \mu \mathrm{E} /\left(\mathrm{m}^{2} \cdot \mathrm{s}\right)\right)$ 最差; 中、高光强条件 下, 样品中均检测到了绿藻与硅藻, 且其所占比例有随培养时间上升的趋势, 光强越高其所占比例越大, 低光强下则未检 测到绿藻与硅藻. 营养盐添加实验中, $N+P$ 添加组的藻类生长状态最优, $\mathrm{P}$ 添加组较好, $N$ 添加组次之, 对照组最差, 说明 在水华早期 $N 、 P$ 均限制藻类生长, 且 $\mathrm{P}$ 的限制作用大于 $\mathrm{N}$; 此外, 还发现营养盐对水华早期藻类的群落结构有一定影响, 添加 $\mathrm{N}$ 或 (和) $\mathrm{P}$ 后, 绿藻与硅藻均被检测到. 从水华早期控制角度看, 降低光强与营养盐 (主要是 $\mathrm{P}$ ) 均能在一定程度上 控制浮游植物生物量及其生长; 采取降低人射光强的措施后可能会出现更耐受低光的蓝澡尤其微囊藻占据优势的情况, 相对低的 $N 、 P$ 营养盐条件亦可能使蓝藻占优势, 若一定程度上升高 $N 、 P$ 营养盐浓度可使硅藻、绿藻逐渐占据优势, 这为 水华早期的选择性控藻提供一定的借鉴意义.

关键词: 光强;营养盐;浮游植物 ; 增殖;群落结构;水华早期;太湖

\section{The physio-ecological characteristics of algae treated with low temperature and light in- tensity under different light intensity, nitrogen and phosphorus concentrations}

\section{ZHOU Qichao ${ }^{1,2}$, XIONG Qian ${ }^{1,3}$, LI Lin $^{1}$, HU Lili ${ }^{1,3}$ \& SONG Lirong ${ }^{1}$}

(1: State Key Laboratory of Freshwater Ecology and Biotechnology, Institute of Hydrobiology, Chinese Academy of Sciences, Wuhan 430072 , P. R. China)

(2: Yunnan Key Laboratory of Pollution Process and Management of Plateau Lake-Watershed, Yunnan Institute of Environmental Science (Kunming China International Research Center for Plateau Lake), Kunming 650034, P. R. China)

(3: University of Chinese Academy of Sciences, Beijing 100049, P. R. China)

Abstract: The impacts of light intensity and nutrients on the growth and composition of phytoplankton(from Lake Taihu) were investigated after a treatment of low temperature $\left(4^{\circ} \mathrm{C}\right)$ and light intensity $\left(5 \mu \mathrm{E} /\left(\mathrm{m}^{2} \cdot \mathrm{s}\right)\right)$. Results showed that growth of algae under moderate light intensity $\left(50 \mu \mathrm{E} /\left(\mathrm{m}^{2} \cdot \mathrm{s}\right)\right)$ was the best followed by high light intensity $\left(200 \mu \mathrm{E} /\left(\mathrm{m}^{2} \cdot \mathrm{s}\right)\right)$ and low light intensity $\left(5 \mu \mathrm{E} /\left(\mathrm{m}^{2} \cdot \mathrm{s}\right)\right)$. Green algae and diatom were detected under moderate and high light intensity, and the proportion of them increased with culturing time and light intensity, however, they were not detected under low light intensity. Meanwhile, nutrients enrichment bioassays were conducted under moderate light intensity to determine the influence of nutrient limitation on phytoplankton growth and structure during early bloom stage. The results indicated that algae grow better with addition of both nitrogen and phosphorus than phosphorus or nitrogen alone and neither successively, indicating that the growth of algae is sensitive to both nitrogen and phosphorus limitation, and is more sensitive to phosphorus in the early stage of blooms. Additionally, nutrients were also found to influence the structure of phytoplankton community in the early stage of bloom, as green algae and diatom were detec-

* 淡水生态与生物技术国家重点实验室项目 (2014FBZ01) 和国家水体污染控制与治理科技重大专项 (2013ZX07102005 ) 联合资助. 2014-11-28 收稿;2015-03-05 收修改稿. 周起超(1985 ), 男, 博士, 工程师; E-mail:qchzhou@ gmail. com. 熊倩(1988～) (并列第一作者), 女, 硕士研究生; E-mail:xiongqian010@ 163. com.

** 通信作者;E-mail:lrsong@ihb. ac. cn. 
ted after introduction of nitrogen or/and phosphorus. These results implied that reducing light intensity and nutrients(mainly phosphorus) will reduce phytoplankton biomass and growth during early bloom stage. However, some cyanobacteria, Microcystis for example, which is low light intensity tolerance, may dominant after reduction of light intensity and nutrients. Green algae and diatom may dominate gradually with increasing the nitrogen and phosphorus concentrations to a certain extent, which provided possible options for selective controlling during early bloom.

Keywords: Light intensity; nutrient; phytoplankton; proliferation; community structure; early stage of bloom; Lake Taihu

在适宜条件下, 富营养化水体中的浮游植物可通过快速增殖而形成藻类水华, 尤以蓝藻水华最为多 见 $^{[1]}$, 近年我国蓝藻水华亦频繁发生, 如太湖 ${ }^{[2]}$, 尤其北部的梅梁湾、竺山湾为蓝藻水华的重灾区 ${ }^{[3]}$. 蓝藻的 生长与水华形成通常分为 4 个阶段, 即休眠、复苏、生长、上浮和聚集 ${ }^{[4]}$, 春季常以复苏和生长为主, 因而可 视为水华早期,期间蓝藻如微囊藻 (Microcystis) 等可逐渐形成优势,进而在夏、秋季成为水华优势类群.

藻类的生长与演替通常是光照、温度、营养盐、生物因子等共同作用的结果 ${ }^{[4]}$. 光照常被认为是浮游植 物生长的限制因子 ${ }^{[5]}$, 与水华发生和持续时间有关 ${ }^{[6]}$, 具有调节浮游植物群落结构的作用 ${ }^{[7-8]}$. 氮、磷营养盐 与藻类生长、群落结构的关系研究较多 ${ }^{[9-13]}$, 而氮磷比与水华形成或演替的因果关系仍存在争议 ${ }^{[4]}$. 然而, 关 于光强、营养盐对水华早期藻类增殖与群落结构影响的研究相对较少.

尽管已有一些关于光强、营养盐对藻类增殖或竞争演替影响的研究 ${ }^{[14-17]}$, 但多以室内培养的 (单一) 藻 种、培养基为实验材料而不足以准确评估野外实际, 如室内培养的藻种或株系不同于原位藻类、或已经过长 期驯化, 培养基与实际水体条件不同等. 因此, 本文研究光强、氮磷营养盐对经低温、低光处理模拟越冬后的 水柱藻类增殖与组成的影响, 旨在为水华早期基于生态因子调控的控藻措施提供借鉴.

\section{1 材料与方法}

\section{1 实验材料}

2012 年 8 月, 分别采集太湖梅梁湾 $\left(31.41^{\circ} \mathrm{N}, 120.19^{\circ} \mathrm{E}\right)$ 水下 $0.5 、 1.7 \mathrm{~m}$ 处水样, 等体积混合后通过 $25^{\#}$ 浮游生物网 $(64 \mu \mathrm{m})$ 过滤浓缩, 以 Whatman $\mathrm{GF} / \mathrm{C}$ 膜过滤的湖水为培养基, 培养于 $4^{\circ} \mathrm{C}$ 冰箱, 光强约 $5 \mu \mathrm{E} /$ $\left(\mathrm{m}^{2} \cdot \mathrm{s}\right)$ 、光周期约 $12 \mathrm{~h}: 12 \mathrm{~h}$, 低温、低光下培养 4 个月 (期间不进行传代) 以模拟越冬备用; 同时, 采集湖水 并通过 GF/C 膜抽滤备用. 低温、低光培养处理后, 蓝藻、绿藻的 PS II 最大光化学量子产量 $\left(F_{\mathrm{v}} / F_{\mathrm{m}}\right)$ 分别为 $0.12 \pm 0.03 、 0.12 \pm 0.02$, 且未检测到硅藻的 $F_{\mathrm{v}} / F_{\mathrm{m}}$ 值; 藻类组成几乎无变化, 处理前、后均为微囊藻属在数 量上占绝对优势, 并伴有伪鱼腥藻属 (Pseudanabaena)、栅藻属 (Scenedesmus)、菱形藻属 (Nitzschia) 等.

\section{2 实验设计}

光强实验中, 将经低温、低光处理的藻种用蒸馏水洗涤 3 次后, 以 $98.4 \mu \mathrm{g} / \mathrm{L}$ 的初始浓度接种于装有 $150 \mathrm{ml}$ 经 $\mathrm{GF} / \mathrm{C}$ 膜过滤湖水 $(\mathrm{N}=2.772 \mathrm{mg} / \mathrm{L}, \mathrm{P}=0.171 \mathrm{mg} / \mathrm{L})$ 的 $250 \mathrm{ml}$ 堆形瓶中, 3 个处理组分别为高光强 组 $\left(200 \mu \mathrm{E} /\left(\mathrm{m}^{2} \cdot \mathrm{s}\right)\right)$ 、中光强组 $\left(50 \mu \mathrm{E} /\left(\mathrm{m}^{2} \cdot \mathrm{s}\right)\right)$ 、低光强组 $\left(5 \mu \mathrm{E} /\left(\mathrm{m}^{2} \cdot \mathrm{s}\right)\right)$.

营养盐实验中, 将过滤湖水稀释作为培养基母液, 分别添加 $\mathrm{NaNO}_{3} 、 \mathrm{~K}_{2} \mathrm{HPO}_{4}$ 或二者均添加用来设置不同 $\mathrm{N} 、 \mathrm{P}$ 浓度的培养基以接近太湖实际, 4 个营养盐处理组分别为对照组 $(\mathrm{N}=0.558 \mathrm{mg} / \mathrm{L}, \mathrm{P}=0.027 \mathrm{mg} / \mathrm{L}) 、 \mathrm{~N}$ 添加组 $(N=2.0 \mathrm{mg} / \mathrm{L}, \mathrm{P}=0.027 \mathrm{mg} / \mathrm{L}) 、 \mathrm{P}$ 添加组 $(\mathrm{N}=0.558 \mathrm{mg} / \mathrm{L}, \mathrm{P}=0.5 \mathrm{mg} / \mathrm{L}) 、 \mathrm{~N}+\mathrm{P}$ 添加组 $(\mathrm{N}=$ $2.0 \mathrm{mg} / \mathrm{L}, \mathrm{P}=0.5 \mathrm{mg} / \mathrm{L})$, 接种同光强实验, 培养光强为 $50 \mu \mathrm{E} /\left(\mathrm{m}^{2} \cdot \mathrm{s}\right)$.

7 个处理组培养条件均为光暗比 $12 \mathrm{~h}: 12 \mathrm{~h}$ 、温度 $25 \pm 1^{\circ} \mathrm{C}$, 每组设 3 个平行, 每 $2 \mathrm{~d}$ 取样 1 次测定相关指 标. 实验所用光源均为可调光强节能灯 ( $20 \mathrm{~W} 、$ cool daylight, Philips).

\section{3 指标测定}

$\mathrm{N} 、 \mathrm{P}$ 浓度测定根据《湖泊富营养化调查规范》 ${ }^{[18]}$. 叶绿素 a ( Chl. a) 浓度常为表征浮游植物生物量的重 要指标, 其测定参照文献 [19], 类胡萝卜素相对含量 (即其与 Chl. a 浓度的比值) 是反映浮游植物抵御高光 强胁迫的指标, 类胡夢卜素浓度测定参照文献 $[20]$. 叶绿素苂光活性是反映藻类光合效率与生理状态的重 要指标, 其值由调制荧光仪 Phyto-PAM 测定, 测定前将样品暗适应 $10 \mathrm{~min}$; 本研究样品中并未观测到甲藻, 故 而该仪器测定的 Brown 只代表硅藻, 并利用光学显微镜鉴定藻类组成 ${ }^{[21]}$. 


\section{4 数据分析}

非参数分析, Nonparametric Tests-2 Independent Samples ( Mann-Whitney U)、Nonparametric Tests-K Independent Samples (Kruskal-Wallis H) 与相关性分析 (Spearman correlation analysis) 均用 SPSS 16.0 软件完成, $P<0.05$ 表示显著相关, $P<0.01$ 表示极显著相关.

\section{2 结果}

\section{1 藻类生长}

实验期间, 经低温、低光处理后, 藻类恢复生长具有较长的调整期. 不同光强的实验结果显示 (图 1a), 相 比高光强组, 中光强组更利于藻类增殖, 而低光强组的 Chl. a 浓度基本呈下降趋势, 3 组间具显著性差异 $(P<0.05)$. 营养盐添加实验结果表明 (图 $1 \mathrm{~b}$ ), 恢复生长过程中 $\mathrm{N} 、 \mathrm{P}$ 浓度均在一定程度上限制藻类增殖, 生 长状态的先后顺序为 $N+P$ 添加组 $>P$ 添加组 $>N$ 添加组 $>$ 对照组, 4 组间具显著性差异 $(P<0.05)$.
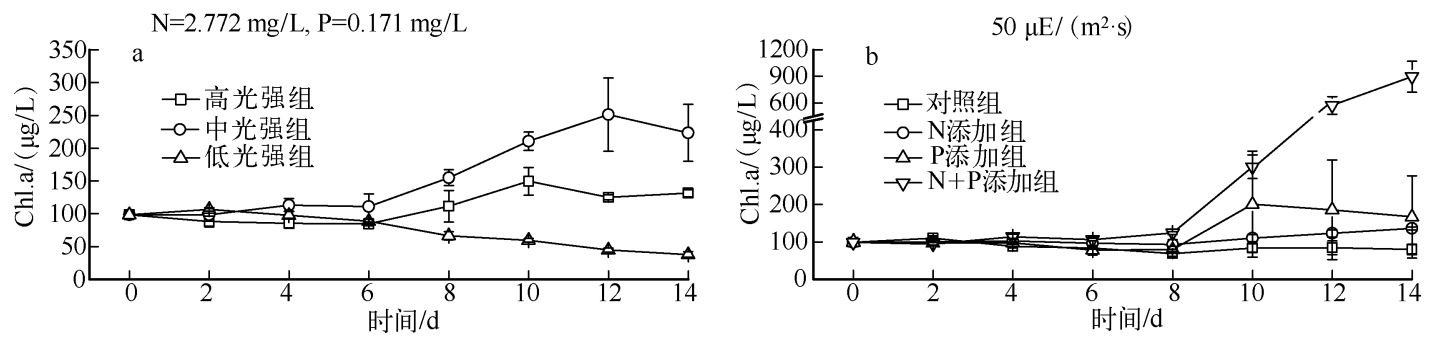

图 1 不同光强 (a) 和营养盐浓度 (b)下的藻类生长曲线

Fig. 1 Growth curves of algae under different light intensities(a) and nutrients concentration(b)

\section{2 藻类类胡夢卜素相对含量变化}

实验期间, 各组藻类的类胡夢卜素相对含量 (图 2) 表明, 除低光强组外, 高光强组、中光强组类胡夢卜素 相对含量均在第 $2 \sim 4 \mathrm{~d}$ 上升, 之后 3 组均相对稳定, 且高光强组类胡夢卜素相对含量显著高于中光强组和 低光强组 $(P<0.05)$. 营养盐添加的实验结果显示, 对照组、 $\mathrm{N}$ 添加组、 $\mathrm{P}$ 添加组从第 $2 \mathrm{~d}$ 开始上升至第 $8 \mathrm{~d}$, 且在第 $12 \sim 14 \mathrm{~d}$ 出现明显下降; $N+P$ 添加组则上升至第 $8 d$ 后即明显下降, 从第 $10 \mathrm{~d}$ 开始保持相对稳定的

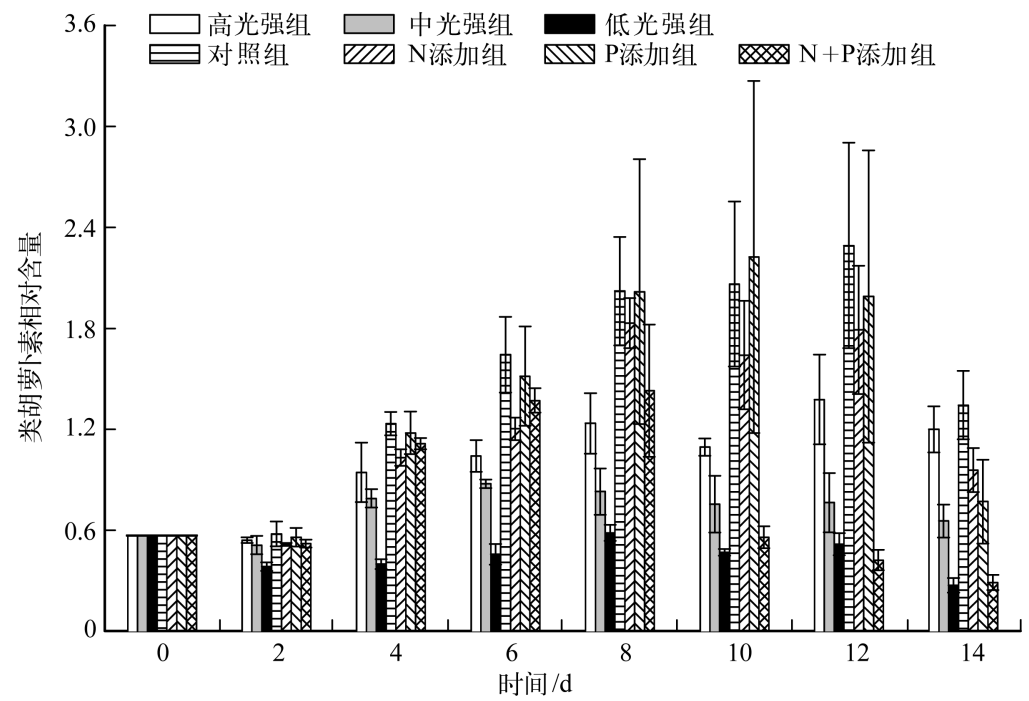

图 2 藻类类胡夢卜素相对含量变化

Fig. 2 Variation of relative content of carotenoids of algae during the experiment 
水平且与低光强组相当; 4 组间, 对照组与 $\mathrm{P}$ 添加组总体含量相当, 且均高于 $\mathrm{N}$ 添加组, 但不具显著性差异 $(P>0.05)$, 该 3 组均显著高于 $\mathrm{N}+\mathrm{P}$ 添加组 $(P<0.05)$.

\section{3 藻类光合活性变化}

实验期间,各处理组藻类 $F_{\mathrm{v}} / F_{\mathrm{m}}$ 变化如图 3 所示: 恢复生长初始第 $2 \mathrm{~d}$, 中光强条件下 (共 5 组) 与 低光强组的藻类活性快速升高, 高光强组 $F_{\mathrm{v}} / F_{\mathrm{m}}$ 在第 $4 \mathrm{~d}$ 出现上升. 光强实验中, 高光强组蓝澡活性衰 弱先于绿藻, 硅藻活性逐渐占优势; 中光强组第 $0 \sim 6 \mathrm{~d}$ 蓝藻活性大于绿藻, 随后蓝藻活性逐渐减弱, 绿 藻活性从第 $10 \mathrm{~d}$ 开始降低, 且第 $10 \mathrm{~d}$ 时出现硅藻活性之后逐渐升高; 低光强组未检测到硅藻活性, 且 蓝藻活性高于绿藻. 营养盐实验中, 相比对照组, $\mathrm{N}$ 添加组未检测到硅藻活性, 蓝藻、绿藻活性均较高; $\mathrm{P}$ 添加组, 蓝藻、绿藻活性于第 $8 \mathrm{~d}$ 开始衰退; $\mathrm{N}+\mathrm{P}$ 添加组从第 $10 \mathrm{~d}$ 起, 蓝藻活性先于绿藻衰弱. 实验 期间, 各处理组初始斜率 $(\alpha)$ 与最大相对电子传递速率 $\left(r E T R_{\text {max }}\right)$ 变化如图 4 所示, 其表现大体与 $F_{\mathrm{v}} / F_{\mathrm{m}}$ 一致.
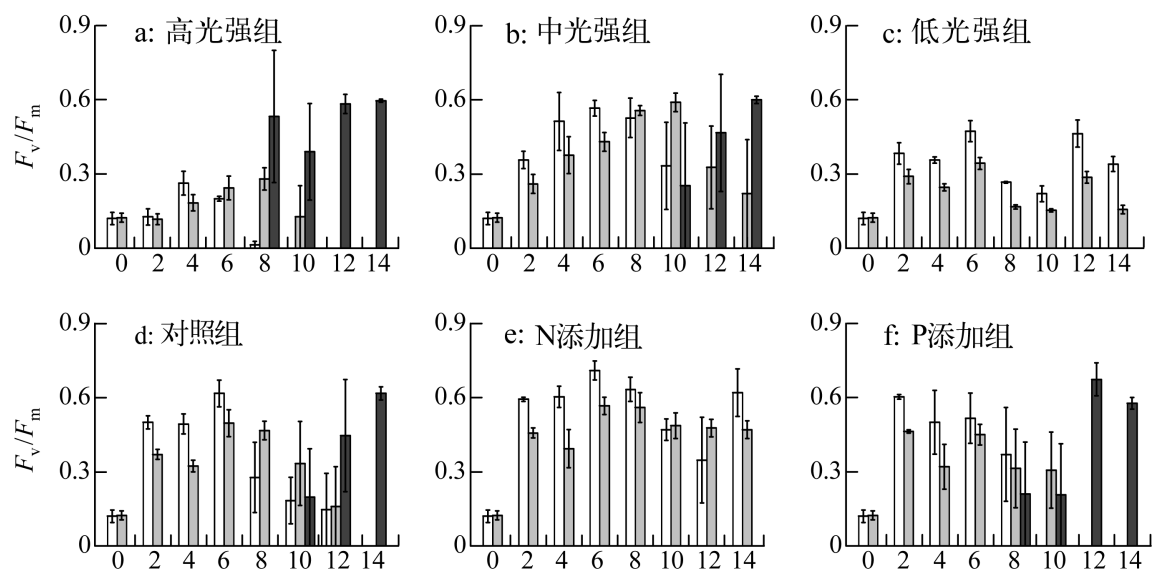

时间/d

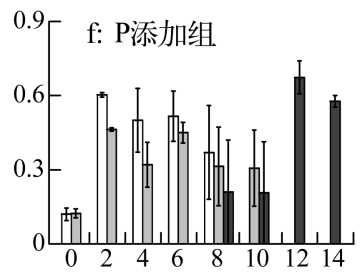

时间/d

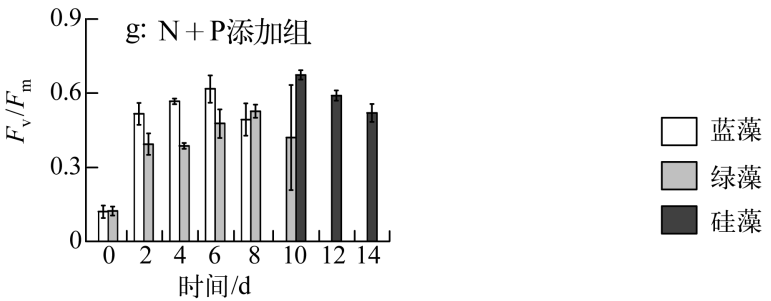

图 3 藻类 PS II 最大光化学量子产量变化

Fig. 3 Variation of $F_{\mathrm{v}} / F_{\mathrm{m}}$ of algae during the experiment

\section{4 藻类组成变化}

实验期间, 各处理组的藻类组成 (各门藻类细胞数所占比例) 变化 (图 5 ) 表明, 蓝藻 (主要为微囊藻) 所 占比例最高, 平均比例均高于 $68 \%$, 且蓝藻所占比例在各处理组的前 $6 \mathrm{~d}$ 均约占近 $100 \%$. 低光强组在整个 实验期间均为蓝藻占优势, 中光强组和高光强组均在第 $8 \mathrm{~d}$ 开始检测出绿藻与硅藻, 且其所占比例有逐渐上 升趋势, 高光强组的比例高于中光强组. 添加 $N 、 P$ 营养盐的实验中, $N$ 添加组从第 $8 \mathrm{~d}$ 开始出现少量的绿藻 与硅藻, 其它 3 组的绿藻所占比例均有升高趋势.

\section{3 讨论}

\section{1 藻类生长}

无论是湖泊生态系统的食物网底层还是水华蓝藻的大量生长, 均受到物质与能量平衡关系的限制 ${ }^{[3,22]}$. 

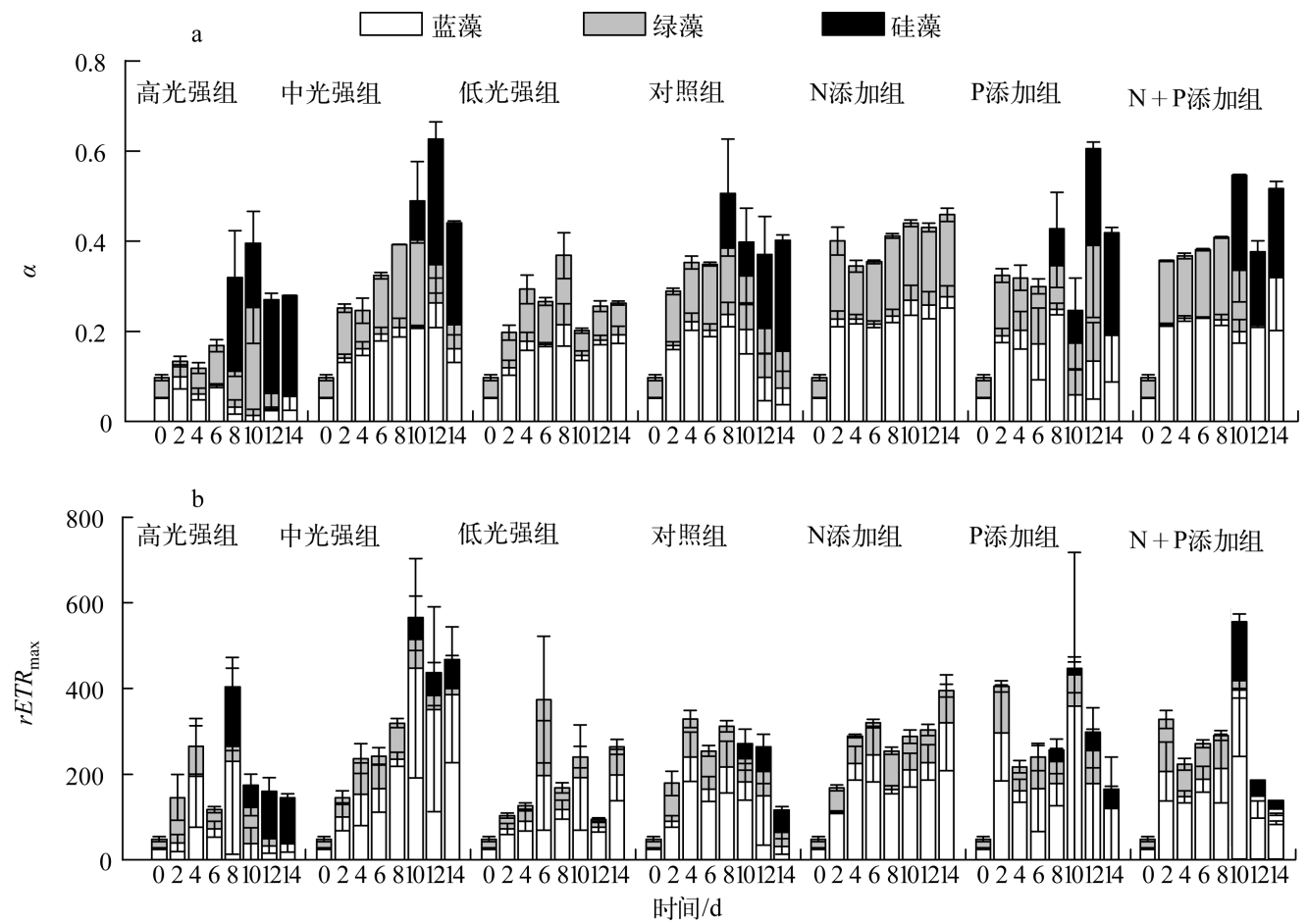

图 4 藻类初始斜率 $(\mathrm{a})$ 与最大相对电子传递速率 $(\mathrm{b})$ 变化

Fig. 4 Variation of $\alpha$ (a) and $r E T R_{\max }$ (b) during the experiment

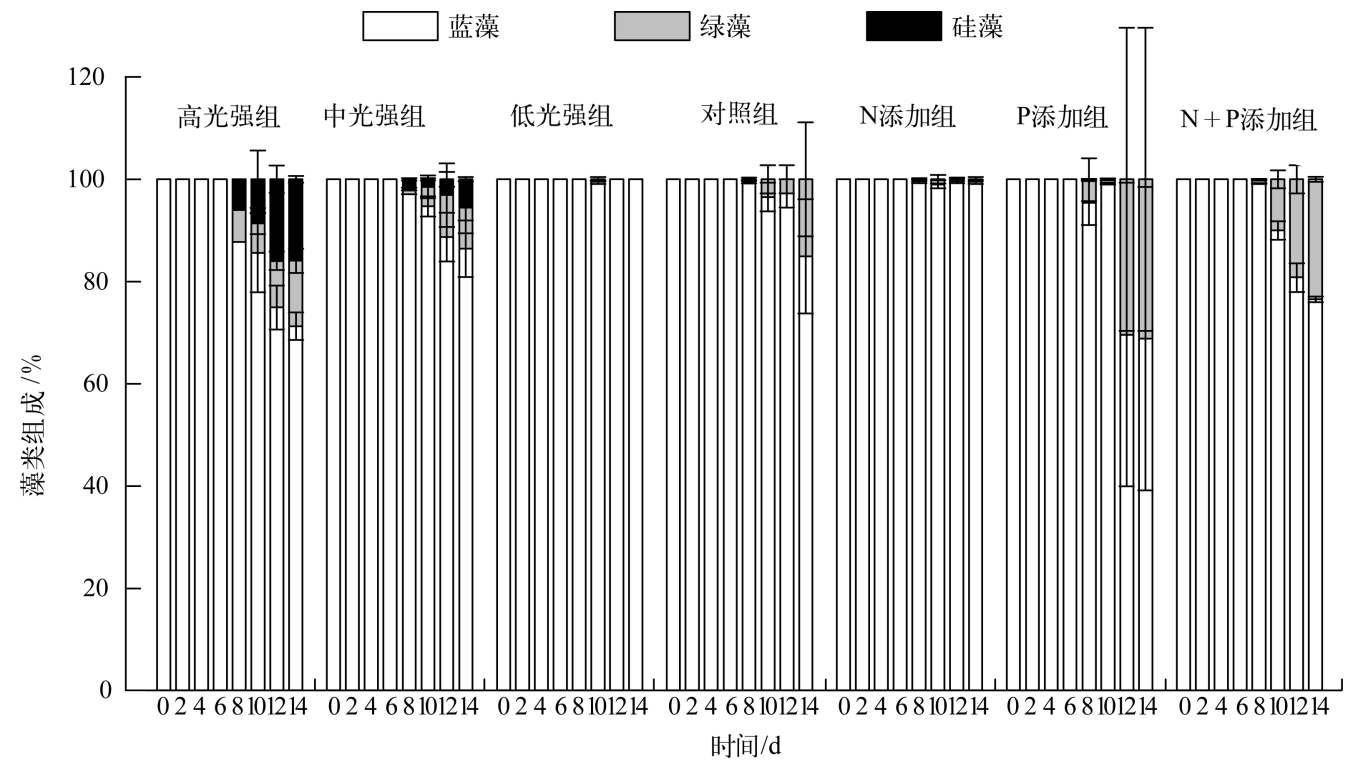

图 5 藻类组成变化

Fig. 5 Variation of algae composition during the experiment

Tomioka 等 ${ }^{[23]}$ 认为人射至水柱中的光强是影响 Kasumigaura 湖中铜绿微囊藻 (Microcystis aeruginosa) 生长的 最重要因子, 营养盐充足与否也很关键 ${ }^{[11-12]}$; 换言之, 虽然温度常被认为是太湖藻类复苏及后续生长的关键 
因子 ${ }^{[3,38]}$, 但在适宜的温度下 $\left(\right.$ 本研究为 $25^{\circ} \mathrm{C}$ ), 藻类复苏至水柱后的生长过程亦常受光强与营养盐的作用. 与此同时, 本研究中无论哪个处理组, 藻类增殖均有 $6 \mathrm{~d}$ 的调整期, 这与低温、低光模拟越冬过程使藻类生理 活性受到一定抑制有关 ${ }^{[24]}$.

光强对藻类生长的影响方面, 光照能引起底泥藻类向水柱中迁移 ${ }^{[25]}$, 且一定光强范围内藻类的生长速 率会随光强的升高而升高 ${ }^{[26-28]}$, 虽然本研究中低光强组的蓝藻 (优势类群) 对光能的利用效率要高于高光强 组 ${ }^{[29-30]}$, 但其总体接受的光能最低, 因此低光强组 $\left(5 \mu \mathrm{E} /\left(\mathrm{m}^{2} \cdot \mathrm{s}\right)\right)$ 藻类生物量无明显增加, 这主要是由光 限制引起的. 高光强组的蓝藻会受到一定程度的光胁迫, 本研究中高光强组 $\left(200 \mu \mathrm{E} /\left(\mathrm{m}^{2} \cdot \mathrm{s}\right)\right)$ PS II 最大光 量子产量的值明显低于其它光强条件, 说明受到一定程度的高光强胁迫 ${ }^{[31-32]}$. 藻类的类胡夢卜素功能主要 为两方面: 一是作为辅助捕光色素 (主要吸收 $400 \sim 550 \mathrm{~nm}$ ) 提高细胞的光合生产, 二是对光氧化的保护作 用,这主要体现在类胡萝卜素可消散来自高度激发的三线态叶绿素 a 或单线态氧的能量; 而提高类胡萝卜 素相对含量是藻类抵御高光强损伤以及维持甚至在一定程度上提高光合作用的一种响应机制 ${ }^{[1,33-34]}$, 故而 本研究高光强条件下的微囊藻仍有一定的增殖. 可见, 光强过高或过低均不利于越冬藻类的复苏生长, 相较 而言中光强较合适. 自然水体中, 光强随深度增加而呈指数下降, 底层水体中易造成微囊藻增殖的光限制、 表层水中则更易造成高光强胁迫, 但微囊藻可通过浮力调节机制运动至合适光强的位置 ${ }^{[35-37]}$. 本研究发现, 相比高光强胁迫, 低光强限制对控制微囊藻增殖及其生物量的效果更明显, 且更易于自然条件下的实际操 作 ${ }^{[38]}$, 如通过遮光等措施人为模拟水面光强低于微囊藻增殖临界光强的条件.

增加营养盐可促进水华早期的藻类生长 ${ }^{[39]}$, 本研究所设的 $50 \mu \mathrm{E} /\left(\mathrm{m}^{2} \cdot \mathrm{s}\right)$ 光强相对利于藻类增殖, 且 $N$ 、 $P$ 浓度参照太湖水华早期的实际情况 ${ }^{[11]}$. 通常浮游植物细胞组成中的氮磷比约为 $16: 1^{[40]}$, 即浮游植物生产同 一单位生物量对 $N$ 的需求量大于 $P$, 虽然其对 $P$ 具有富集积累作用 ${ }^{[5,39]}$, 但低温低光处理后或已使其消耗, 故 而本研究中 $\mathrm{N}$ 添加组藻类的生长状况劣于 $\mathrm{P}$ 添加组, 即使蓝藻的叶绿素苂光值略高于 $\mathrm{P}$ 添加组; 相同光强下 若营养盐相对缺乏可导致光能相对过剩, $\mathrm{N}+\mathrm{P}$ 添加组的藻类类胡萝卜素相对含量低且 $F_{\mathrm{v}} / F_{\mathrm{m}}$ 值高, 说明相较 而言该处理组藻类受胁迫小, 故而其生长状况最优, 与相关报道一致 ${ }^{[41]}$; 但对照组生长状况最劣, $\mathrm{N}+\mathrm{P}$ 添加组 的生长状况明显优于中光强组 (中光强组的 $N$ 浓度高于 $N+P$ 添加组、P 浓度低于 $N+P$ 添加组), 这说明水华 早期 $\mathrm{P}$ 的限制作用大于 $\mathrm{N}$, 与 $\mathrm{P}$ 是春季太湖浮游植物生长的主要限制因子的报道吻合 ${ }^{[11-12]}$. 本研究还发现, 光 限制时 (低光强组) 浮游植物的生长劣于光照充足但 N、P 浓度相对缺乏的处理组 (即对照组), 说明光限制时 光强为浮游植物生长的第一限制因子, 这与光强不足时营养盐会变得相对富足的报道一致 ${ }^{[22,4243]}$.

因此, 若从水华早期控藻的角度看, 降低光强与营养盐 (主要是 P) 均能在一定程度上控制浮游植物生 物量及其生长.

\section{2 藻类组成}

虽然温度被认为在影响太湖浮游植物组成中扮演着重要作用 ${ }^{[44]}$, 生物因子亦被认为是太湖梅梁湾春夏 之交浮游植物演替的主要影响因子 ${ }^{[10]}$, 但本研究发现光强与营养盐在水华早期对浮游植物的组成变化均起 到了一定作用. 本研究低光强处理组基本未检测到绿藻、硅藻的光合活性或生物量, 中、高光强处理组则在 实验中、后期出现了硅藻、绿藻, 且其所占比例有随时间上升的趋势, 光强越高其所占比例越大, 这与绿藻、 硅藻比蓝藻 (微囊藻) 更喜好相对高的光强吻合 ${ }^{[15,33,45]}$; 自然条件下, 太湖梅梁湾中蓝藻晚于硅藻、绿藻到夏 季才占据优势 ${ }^{[10,46]}$, 虽然这有温度逐渐升高的原因, 但也可能与风场导致的藻类堆积及悬浮物浓度升高进 而影响水下光场变化有关 ${ }^{[43,46]}$.

据报道, 太湖河口硅藻生物量密集与相对高的 N、P 浓度有关 ${ }^{[47]}$, 太湖冬、春季营养盐的增加可提高硅藻的竞 争优势 ${ }^{[48]}$; 同时,诸多研究均表明在相对高的 N、P 浓度下绿藻(栅藻) 较蓝藻(微囊藻) 更易形成竞争优势(24～ $\left.28^{\circ} \mathrm{C}\right)^{[49-51]}$. 本研究各处理组均在实验中、后期观测到绿藻、硅藻, 说明营养盐对水华早期藻类的群落结构有一定影 响; 而期间绿藻相对硅藻更占优势, 可能是因为绿藻生长的最适温度 $\left(20 \sim 25^{\circ} \mathrm{C}\right)$ 高于硅藻的最适温度 $(14 \sim$ $\left.18^{\circ} \mathrm{C}\right)^{[48]}$, 而本研究所设温度为 $25^{\circ} \mathrm{C}$, 蓝藻( 微囊藻) 生长的最适温度则更高, 故而蓝藻所占比例一直最高.

因此, 若从水华早期控制角度看, 采取降低人射光强的措施虽可降低藻类生物量, 但可能会出现更耐低 光的蓝藻尤其微囊藻占据优势的情况; 若不考虑温度, 相对低的 N、P 营养条件亦可能使蓝藻占优势, 若一定 程度上升高 $\mathrm{N} 、 \mathrm{P}$ 浓度可使硅藻、绿藻逐渐占据优势. 


\section{4 参考文献}

[ 1 ] Paerl HW, Fulton RS, Moisander PH et al. Harmful freshwater algal blooms, with an emphasis on cyanobacteria. The Scientific World Journal, 2001, (1) : 76-113.

[2] 秦伯强. 太湖生态与环境若干问题的研究进展及其展望. 湖泊科学, 2009, 21 (4): 445-455. DOI 10. 18307/ 2009. 0401.

[ 3 ] 孔繁翔, 马荣华, 高俊峰等. 太湖蓝藻水华的预防、预测和预警的理论与实践. 湖泊科学, 2009,21(3):314-328. DOI 10. $18307 / 2009.0302$.

[ 4 ] 孔繁翔,高 光. 大型浅水富营养化湖泊中蓝藻水华形成机理的思考. 生态学报, 2005,25(3):589-595.

[ 5 ] Darley WM. Algal biology : A physiological approach. In: Wilkinson JF ed. Basic microbiology, Vol. 9. Oxford London: Blackwell Scientific Publication, 1982.

[ 6 ] Zhang M, Duan H, Shi X et al. Contributions of meteorology to the phenology of cyanobacterial blooms: Implications for future climate change. Water Research, 2012, 46(2) : 442-452.

[ 7 ] Havens KE, Phlips EJ, Cichra MF et al. Light availability as a possible regulator of cyanobacteria species composition in a shallow subtropical lake. Freshwater Biology, 1998, 39(3) : 547-556.

[ 8 ] Litchman E. Population and community responses of phytoplankton to fluctuating light. Oecologia, 1998, 117 (1/2): 247-257.

[ 9 ] Paerl HW, Fulton III RS. Ecology of harmful cyanobacteria. In: Graneli E, Turner JT eds. Ecology of harmful algae. Berlin: Springer, 2006.

[10] Ke Z, Xie P, Guo L. Controlling factors of spriner phytoplankton succession in Lake Taihu (Meiliang Bay, China). Hydrobiologia, 2008, 607: 41-49.

[11] Xu H, Paerl HW, Qin B et al. Nitrogen and phosphorus inputs control phytoplankton growth in eutrophic Lake Taihu, China. Limnology and Oceanography, 2010, 55(1): 420-432.

[12] Paerl HW, Xu H, McCarthy MJ et al. Controlling harmful cyanobacterial blooms in a hyper-eutrophic lake( Lake Taihu, China): The need for a dual nutrient (N \& P) management strategy. Water Research, 2011, 45(5): 1973-1983.

[13] Dai G, Shang J, Qiu B. Ammonia may play an important role in the succession of cyanobacterial blooms and the distribution of common algal species in shallow freshwater lakes. Global Change Biology, 2012, 18(5) : 1571-1581.

[14] Huisman J. Population dynamics of light-limited phytoplankton: Microcosm experiments. Ecology, 1999, 80 (1): 202-210.

[15] Huisman J, Jonker RR, Zonneveld C et al. Competition for light between phytoplankton species: Experimental tests of mechanistic theory. Ecology, 1999, 80(1) : 211-222.

[16] Passarge J, Hol S, Escher M et al. Competition for nutrients and light: Stable coexistence, alternative stable states, or competitive exclusion? Ecological Monographs, 2006, 76(1): 57-72.

[17] 袁丽娜, 宋 炜, 肖 琳等. 多环境因素全面正交作用对铜绿微囊藻生长的效应研究. 南京大学学报: 自然科学, $2008, \mathbf{4 4}(4): 408-414$.

[18］金相灿, 屠清瑛. 湖泊富营养化调查规范:第 2 版. 北京: 中国环境科学出版社, 1990.

[19] Mitchell BG, Kiefer DA. Determination of absorption and fluorescence excitation spectra for phytoplankton. In: HolmHansen O, Bolis L, Gilles R eds. Marine phytoplankton and productivity. Berlin: Springer-Verlag, 1984: 157-169.

$[20]$ Починок $\Phi H$ 著. 荆家海, 丁钟荣译. 植物生物化学分析方法. 北京:科学出版社, 1981 :255-258.

[21] 胡鸿钧,魏印心. 中国淡水藻类——系统、分类及生态. 北京: 科学出版社, 2006 .

[22] Sterner RW, Elser JJ, Fee EJ et al. The light: nutrient ratio in lakes: The balance of energy and materials affects ecosystem structure and process. American Naturalist, 1997, 150(6) : 663-684.

[23] Tomioka N, Imai A, Komatsu K. Effect of light availability on Microcystis aeruginosa blooms in shallow hypereutrophic Lake Kasumigaura. Journal of Plankton Research, 2011, 33(8) : 1263-1273.

[24] 万 能,汤 俊, 宋立荣. 微囊藻休眠体复苏机制的研究进展. 水生态学杂志, 2010,3(4): 113-117.

[25] Tsujimura S, Tsukada H, Nakahara H et al. Seasonal variations of Microcystis populations in sediments of Lake Biwa, Japan. Hydrobiologia, 2000, 434(1/2/3) : 183-192.

[26] Yang Z, Geng L, Wang W et al. Combined effects of temperature, light intensity, and nitrogen concentration on the growth and polysaccharide content of Microcystis aeruginosa in batch culture. Biochemical Systematics and Ecology, 2012, 41: 130-135. 
[27] Carneiro RL, dos Santos MEV, Pacheco ABF et al. Effects of light intensity and light quality on growth and circadian rhythm of saxitoxins production in Cylindrospermopsis raciborskii (Cyanobacteria). Journal of Plankton Research, $2009, \mathbf{3 1}$ (5) : $481-488$.

[28] Xiao Y, Gan N, Liu J et al. Heterogeneity of buoyancy in response to light between two buoyant types of cyanobacterium Microcystis. Hydrobiologia, 2012, 679(1) : 297-311.

[29] Zhang M, Shi X, Yu Y et al. The acclimative changes in photochemistry after colony formation of the cyanobacteria Microcystis aeruginosa. Journal of Phycology, 2011, 47(3) : 524-532.

[30] Perkins RG, Mouget JL, Lefebvre S et al. Light response curve methodology and possible implications in the application of chlorophyll fluorescence to benthic diatoms. Marine Biology, 2006, 149(4) : 703-712.

[31] 韩博平, 韩志国, 付 翔. 藻类光合作用机理与模型. 北京:科学出版社, 2003 .

[32] Zhang M, Kong F, Wu X et al. Different photochemical responses of phytoplankters from the large shallow Taihu Lake of subtropical China in relation to light and mixing. Hydrobiologia, 2008, 603(1) : 267-278.

[33] Xu K, Jiang H, Juneau P et al. Comparative studies on the photosynthetic responses of three freshwater phytoplankton species to temperature and light regimes. Journal of Applied Phycology, 2012, 24(5) : 1113-1122.

[34] Paerl HW. Cyanobacterial carotenoids: Their roles in maintaining optimal photosynthetic production among aquatic bloom forming genera. Oecologia, 1984, 61(2) : 143-149.

[35] Bormans M, Sherman BS, Webster IT. Is buoyancy regulation in cyanobacteria an adaptation to exploit separation of light and nutrients? Marine and Freshwater Research, 1999, 50(8) : 897-906.

[36] 成慧敏,邱保胜. 蓝藻的伪空泡及其对蓝藻在水体中垂直分布的调节. 植物生理学通讯,2006,42(5):974-980.

[37] Wallace BB, Bailey MC, Hamilton DP. Simulation of vertical position of buoyancy regulating Microcystis aeruginosa in a shallow eutrophic lake. Aquatic Sciences, 2000, 62(4) : 320-333.

[38] Zhou QC, Chen W, Shan K et al. Influence of sunlight on the proliferation of cyanobacterial blooms and its potential applications in Lake Taihu, China. Journal of Environmental Sciences, 2014, 26(3) : 626-635.

[39] Jia YH, Dan J, Zhang M et al. Growth characteristics of algae during early stages of phytoplankton bloom in Lake Taihu, China. Journal of Environmental Sciences, 2013, 25 (2) : 254-261.

[40] Reynolds CS. Ecology of phytoplankton. In: Usher M, Saunders D, Peet S et al eds. Ecology, biodiversity and conservation. New York: Cambridge University Press, 2006.

[41] Elser JJ, Marzolf ER, Goldman CR. Phosphorus and nitrogen limitation of phytoplankton growth in the freshwaters of North America: A review and critique of experimental enrichments. Canadian Journal of Fisheries and Aquatic Sciences, 1990 , 47(7): 1468-1477.

[42] Havens KE, Steinman AD, Hwang SJ. Phosphorus uptake by plankton and periphyton in relation to irradiance and phosphate availability in a subtropical lake (Lake Okeechobee, Florida, USA). Archiv für Hydrobiologie, 2001, 151 ( 2) : $177-201$.

[43] Duhamel S, Bjoerkman KM, Karl DM. Light dependence of phosphorus uptake by microorganisms in the subtropical North and South Pacific Ocean. Aquatic Microbial Ecology, 2012, 67(3) : 225-238.

[44] Chen YW, Qin BQ, Teubner K et al. Long-term dynamics of phytoplankton assemblages: Microcystis-domination in Lake Taihu, a large shallow lake in China. Journal of Plankton Research, 2003, 25(4) : 445-453.

[45] Coles JF, Jones RC. Effect of temperature on photosynthesis-light response and growth of four phytoplankton species isolated from a tidal freshwater river. Journal of Phycology, 2000, 36(1): 7-16.

[46] 孟顺龙, 陈家长, 胡庚东等. 2008 年太湖梅梁湾浮游植物群落周年变化. 湖泊科学, 2010, 22 (4): 577-584. DOI 10. 18307/2010.0415.

[47] 刘 霞, 陆晓华, 陈宇炜. 太湖浮游硅藻时空演化与环境因子的关系. 环境科学学报,2012,32(4):821-827.

[48] 吴 攀, 邓建明, 秦伯强等. 水温和营养盐增加对太湖冬、春季节藻类生长的影响. 环境科学研究, 2013, 26 (10): 1064-1071.

[49] 许 海, 杨林章, 刘兆普. 铜绿微囊藻和斜生栅藻生长的氮营养动力学特征. 环境科学研究, 2008,21(1):69-73.

[50] 许 海, 杨林章, 茅 华等. 铜绿微囊藻、斜生栅藻生长的磷营养动力学特征. 生态环境, 2006,15(5):921-924.

[51] 万 蕾, 朱 伟, 赵联芳. 氮磷对微囊藻和栅藻生长及竞争的影响. 环境科学, 2007,28(6):1230-1235. 